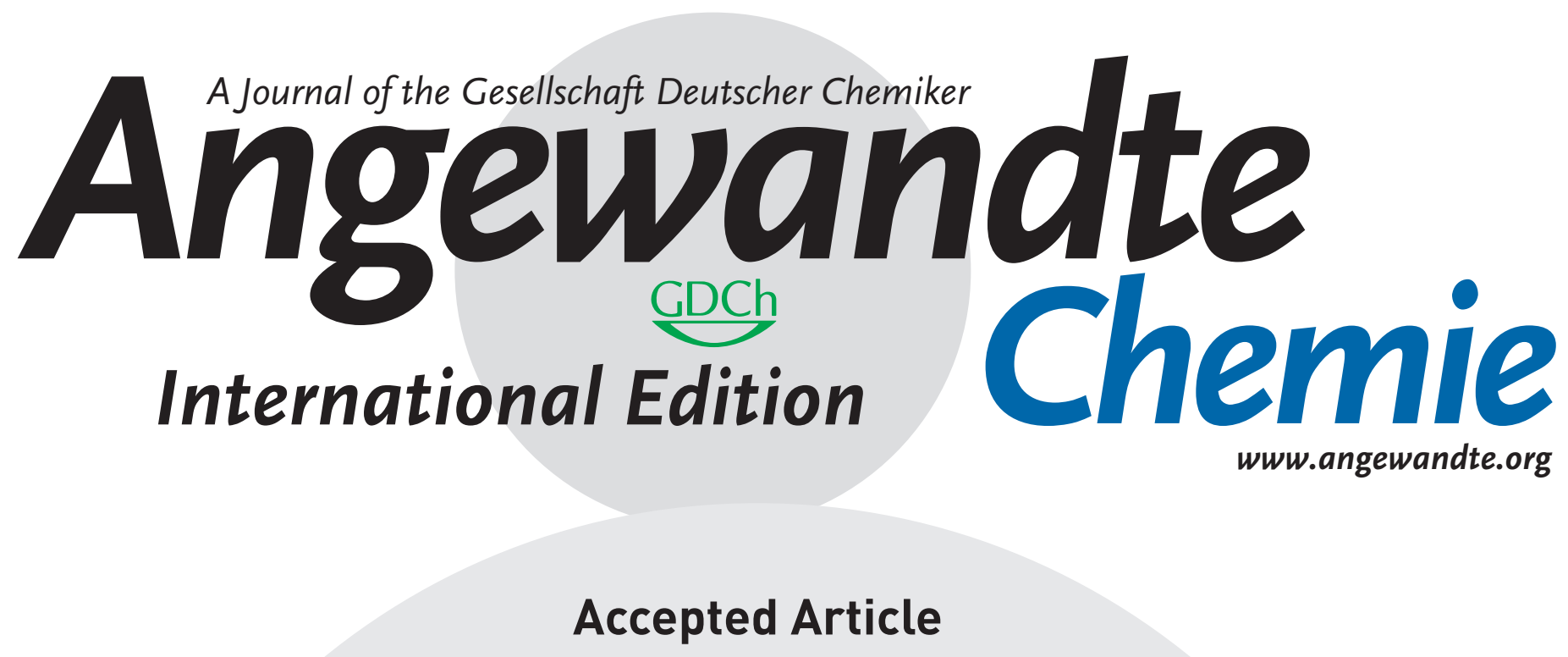

Title: Biocatalytic, Intermolecular C-H Bond Functionalization for the Synthesis of Enantioenriched Amides

Authors: Soumitra Athavale, Shilong Gao, Zhen Liu, Sharath Chandra Mallojjala, Jennifer Hirschi, and Frances H. Arnold

This manuscript has been accepted after peer review and appears as an Accepted Article online prior to editing, proofing, and formal publication of the final Version of Record (VoR). This work is currently citable by using the Digital Object Identifier (DOI) given below. The VoR will be published online in Early View as soon as possible and may be different to this Accepted Article as a result of editing. Readers should obtain the VoR from the journal website shown below when it is published to ensure accuracy of information. The authors are responsible for the content of this Accepted Article.

To be cited as: Angew. Chem. Int. Ed. 10.1002/anie.202110873

Link to VoR: https://doi.org/10.1002/anie.202110873 


\title{
Biocatalytic, Intermolecular C-H Bond Functionalization for the Synthesis of Enantioenriched Amides
}

\author{
Soumitra V. Athavale ${ }^{\dagger[a]}$, Shilong Gao ${ }^{\dagger[a]}$, Zhen Liu ${ }^{[a]}$, Sharath Chandra Mallojjala ${ }^{[b]}$, Jennifer S. \\ Hirschi $^{*[b]}$ and Frances H. Arnold*[a]
}

[a] Dr. S. V. Athavale, S. Gao, Dr. Z. Liu, Prof. F. H. Arnold

Division of Chemistry and Chemical Engineering,

California Institute of Technology,

1200 East California Boulevard, MC 210-41, Pasadena, California 91125, United States.

E-mail: frances@cheme.caltech.edu

[b] Dr. S. C. Mallojjala, Prof. J. S. Hirschi

Department of Chemistry,

Binghamton University,

Binghamton, New York, 13902, United States

E-mail: jhirschi@binghamton.edu

[†] These authors contributed equally.

Supporting information for this article is given via a link at the end of the document

\begin{abstract}
Directed evolution of heme proteins has opened access to new-to-nature enzymatic activity that can be harnessed to tackle synthetic challenges. Among these, reactions resulting from active site iron-nitrenoid intermediates present a powerful strategy to forge $\mathrm{C}-\mathrm{N}$ bonds with high site- and stereoselectivity. Here we report a biocatalytic, intermolecular benzylic $\mathrm{C}-\mathrm{H}$ amidation reaction operating at mild and scalable conditions. With hydroxamate esters as nitrene precursors, feedstock aromatic compounds can be converted to chiral amides with excellent enantioselectivity (up to $>99 \%$ ee) and high yields (up to $87 \%$ ). Kinetic and computational analysis of the enzymatic reaction reveals rate-determining nitrenoid formation followed by stepwise hydrogen atom transfer-mediated $\mathrm{C}-\mathrm{H}$ functionalization.
\end{abstract}

From constituting the building blocks of life to appearing ubiquitously in natural products, therapeutics, polymers and synthetic materials, amides are arguably the most privileged among the nitrogen-containing functional groups. Nearly onefourth of marketed drugs and about two-thirds of drug candidates bear at least one amide bond. ${ }^{[1,2]}$ Amine acylation, the principal method for amide synthesis, is the most common reaction performed in the pharmaceutical industry. ${ }^{[2]}$ However, the approach is fundamentally limited by its reliance on the availability of oxidized precursors (enantioenriched amines) as reactants. Likewise, since acylation with amide coupling reagents produce stoichiometric byproducts and possess poor atom economy, alternative methods to synthesize amides have been recognized as a pressing challenge in green chemistry. ${ }^{[3]}$ Alternatively, access to amides can be envisaged through a direct $\mathrm{C}-\mathrm{H}$ amidation reaction (Figure $1 \mathrm{~A}$ ). Selective $\mathrm{C}-\mathrm{H}$ functionalization an enduring frontier for organic synthesis - offers strategies to promote inert starting materials to useful intermediates, streamline synthesis by using abundant synthons, and at its pinnacle allow precise alteration of complex molecules. ${ }^{[4,5]}$ Here we report that enzymatic $\mathrm{C}\left(\mathrm{sp}^{3}\right)-\mathrm{H}$ functionalization of benzylic positions can provide highly enantioenriched amides from unfunctionalized starting materials. (Figure 1C).

The paradigm to achieve nitrene-transfer mediated, asymmetric $\mathrm{C}-\mathrm{H}$ amidation was recently pioneered by Chang and

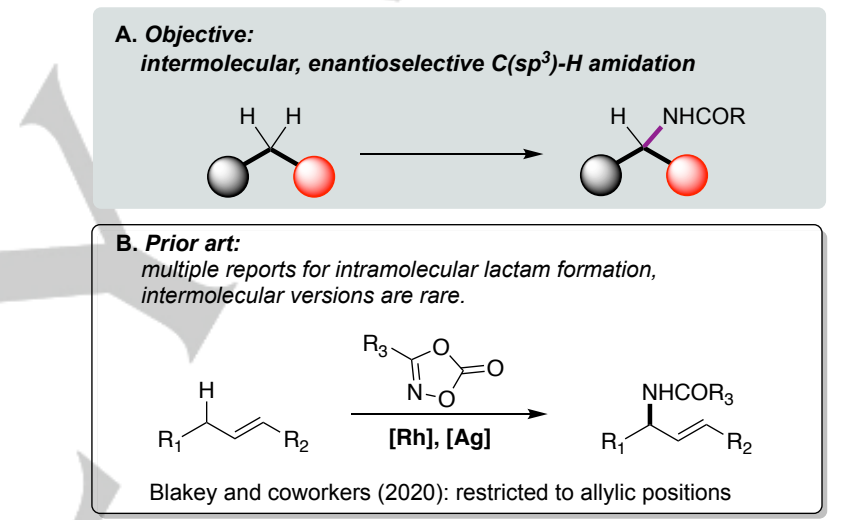

C. This work: biocatalytic benzylic amidation

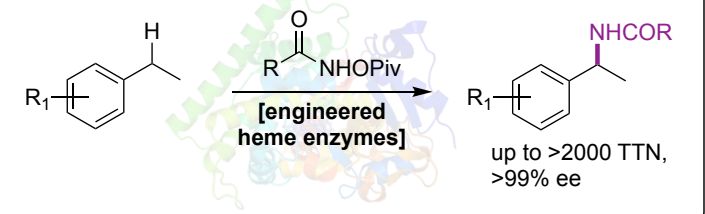

Figure 1: Objectives, state of the art and new findings. (A) intermolecular, enantioselective $\mathrm{C}-\mathrm{H}$ amidation. (B) Previous examples. (C) New findings from this study.

coworkers with the introduction of dioxozolone derivatives as nitrene precursors. ${ }^{[6-8]}$ In the presence of an appropriate organometallic catalyst (typically $\mathrm{Rh}, \mathrm{Ru}$, Ir, or $\mathrm{Co}$ ), the resulting acyl(metal)nitrenoid species can undergo enantioselective $\mathrm{C}-\mathrm{H}$ insertion reactions to form amides. ${ }^{[9]}$ This strategy has largely been confined to intramolecular lactam formation ${ }^{[10-16]}$ and some cases of $\mathrm{C}\left(\mathrm{sp}^{2}\right)-\mathrm{H}$ functionalization. ${ }^{[17,18]}$ Only three enantioselective, intermolecular versions have been reported for $\mathrm{C}\left(\mathrm{sp}^{3}\right)-\mathrm{H}$ amidation. Two, by Matsunaga and coworkers, are restricted to thioamides and quinoline substrates as directing groups. ${ }^{[19,20]}$ The third, more general report by Blakey and coworkers utilizes chiral Rh-indenyl catalysts to amidate allylic positions (Figure 1B). ${ }^{[21]}$

Over the last decade, heme enzymes housing the Feporphyrin cofactor have emerged as efficient biocatalysts for 

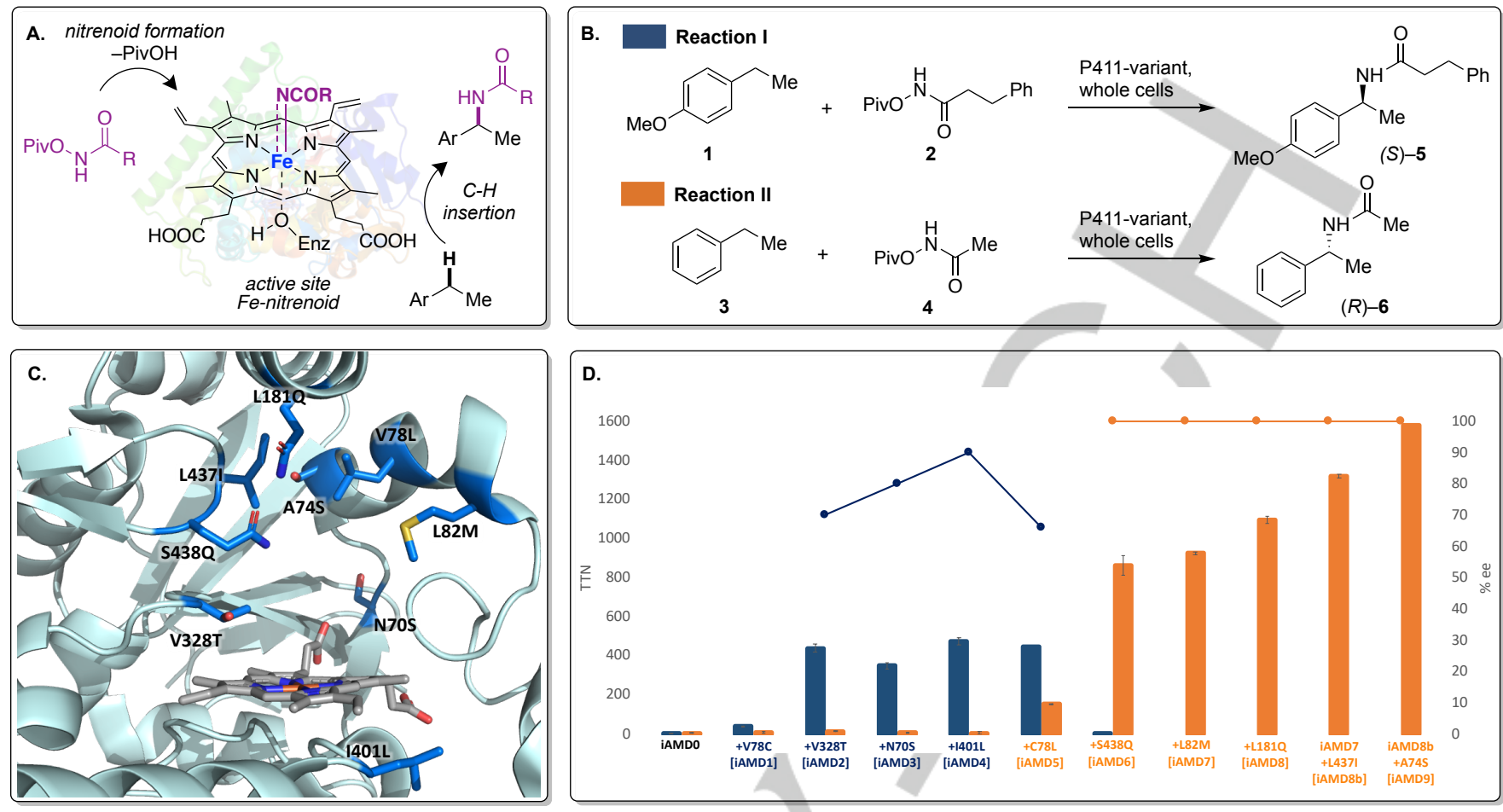

Figure 2: Directed evolution for enzymatic, intermolecular amidation. (A) Hypothesis of the putative reaction pathway (Piv = pivoloyl). (B) Model amidation reactions I and II. (C) Active site model of iAMD9 (based on crystal structure from PDB ID: 5ucw ${ }^{[24}$ ) with mutated residues highlighted. (D) Directed evolution of amidation activity for reaction I (blue) and reaction II (orange). For each variant, bars indicate total turnover numbers (TTNs) for the whole-cell reactions, while circles indicate enantiomeric excess (ee) of product (S)-5 (blue) and $(R)-6$ (orange). Refer to Supplementary Information for experimental details.

carbene and nitrene transfer reactions. ${ }^{[22,23]}$ The active site of P411 enzymes (which bear a Ser in place of Cys for axial coordination to the heme iron atom) can be evolved to stabilize and direct the reactivity of $\mathrm{Fe}$-nitrenoid species to $\mathrm{C}-\mathrm{H}$ insertion and olefin functionalization reactions. ${ }^{[24-26]}$ On this basis, the hypothesis that an active-site acyl Fe-nitrenoid species can be generated and harnessed for an intermolecular $\mathrm{C}-\mathrm{H}$ amidation reaction seemed compelling. Unlike previously reported $\mathrm{Fe}-$ sulfonyl-nitrenoid and unprotected Fe-nitrenoid species, acyl(metal)nitrenes are vulnerable to deleterious, Curtius-type rearrangement processes to make unwanted isocyanate products that add a further challenge to reaction development. ${ }^{[16,27]}$

Hydroxylamine derivatives have been used successfully to generate metal ${ }^{[28-30]}$ and active site nitrenoid species. ${ }^{[25,26]}$ Thus, we hypothesized that pivalate esters of hydroxamic acids, through the loss of pivalic acid, may generate an active site Fe-acylnitrenoid intermediate that can engage in a formal intermolecular $\mathrm{C}-\mathrm{H}$ insertion reaction (Figure 2A). Two transformations were selected as models (Figure 2B): (i) reaction I: benzylic amidation of 4-ethylanisole (1) with 3-phenyl- $N$-(pivaloyloxy)propanamide (2) and, (ii) reaction II: benzylic amidation of ethylbenzene (3) with $\mathrm{N}$-(pivaloyloxy)acetamide (4). The reactions were screened with a panel of engineered $\mathrm{P} 411$ enzymes in whole-cell reactions. Only one variant, henceforth called $i A M D 0$, provided the amidation product, detectable by mass spectroscopy at trace yields $(<1 \%)$ and trace total turnover numbers ( $<10$ TTN). Directed evolution has allowed development of efficient enzymes even from such low activities. ${ }^{[31]}$ Reaction I was chosen for the evolution campaign with the hypothesis that active site modification to accept these larger substrates may simultaneously predispose the evolved enzymes toward smaller reactants (like $\mathbf{3}$ and $\mathbf{4}$ ) rather than vice versa.

Directed evolution involved screening sequential rounds of site-saturation mutagenesis (SSM, one site at a time) at residues close to the heme cofactor in the active site as well as in flexible loop regions of the protein (Figure 2C). SSM mutant libraries were screened for improved activity in whole-cell, 96-well reactions under anaerobic conditions. The crystal structure of a closely related P411 enzyme (PDB ID: 5ucw) was inspected to identify prospective target sites. ${ }^{[24]}$ Four rounds of SSM and screening yielded variant $i A M D 4$ incorporating mutations $\mathrm{V} 78 \mathrm{C}$, $\mathrm{V} 328 \mathrm{~T}$, N70S, and $1401 \mathrm{~L}$, which catalyzed the amidation reaction I with $\sim 475$ TTN and provided (S)-configured amide (5) with an enantiomeric enrichment (ee) of $90 \%$ (Figure 2D). The boost in activity was almost entirely a result of the mutation at position 328 , which lies on a loop close to the distal heme pocket (Figure 2C). Mutations at sites 70 and 401 contributed to improving enantioselectivity. Note that previous reports of intermolecular enzymatic nitrene $\mathrm{C}-\mathrm{H}$ functionalization are restricted to delivering only the $(R)$-configured product. ${ }^{[24,25]}$ 2-Phenylethanamine, the expected Curtius-type rearrangement product of the acyl nitrenoid derived from 2, was not detected. However, this lineage showed no improvement in catalyzing reaction II. Poor activity was also noted in the reaction between $\mathbf{1}$ and $\mathbf{4}$. The hypothesis that these enzymes will be predisposed toward amidation with smaller substrates thus proved to be too simplistic. Hence, reaction II was chosen for subsequent rounds of directed evolution. The C78L mutant that confers moderate activity with reaction II maintains activity with reaction $\mathrm{I}$, although a significant drop in enantioselectivity $(66 \%$ ee) is seen for the formation of $\mathbf{5}$. 
Mutation $\mathrm{S} 438 \mathrm{Q}$ in variant $i A M D 6$ amplified activity for reaction II by $\sim 10$ fold, but at the cost of nearly complete abolishment of activity for reaction I. With variant $i A M D 6$, amide $(R)-6$ is obtained with exceptional enantioselectivity (>99\% ee). This divergence in activities for the two model reactions as well as the opposite configurations of the respective product enantiomers highlights the incompatibility of $\mathbf{2}$ and $\mathbf{4}$ to function in the same active site in this lineage. Further rounds of SSM provided improvements in TTNs while maintaining high enantioselectivity. The evolution campaign concluded with variant iAMD9, which provided $(R)-6$ with 1580 TTN, an analytical yield of $57 \%$ and with $>99 \%$ ee. This value compares favorably with previous $\mathrm{C}-\mathrm{H}$ nitrene insertion reactions with $\mathrm{P} 411$ enzymes ( 300-700 TTN for benzylic sulfonamidation and primary amination), ${ }^{[24,25]}$ but is lower than that seen for carbene $\mathrm{C}-\mathrm{H}$ insertion (upto 3750 TTN). ${ }^{[31]}$ Constitutional isomers arising from competing functionalization of the primary carbon or the aromatic ring were not detected.

To gauge substrate scope, the evolved lineage was tested with various substituted ethylbenzene and aromatic derivatives (Figure 3). Methyl substitution at para-, ortho-, and meta-position (7-9) was tolerated. Naphthyl derivative (10), five- and sixmembered rings $(\mathbf{1 1}, \mathbf{2 1})$, and propylbenzenes were competent substrates $(12,13)$. Halogenated substrates (14-17) gave moderate to low TTNs (215-1000), consistent with the trend of electron-rich substrates performing better than electron-poor ones. The use of $\mathrm{N}$-(pivaloyloxy)propionamide and methyl (pivaloyloxy)carbamate as nitrene precursors allowed the enzymatic synthesis of ethylamide (18) and methylcarbamate (19) derivatives, respectively. With the exception of the indenyl product (11), exquisite enantioselectivity $(>99 \%$ ee, minor enantiomer not detected) was seen in all other cases. Variant iAMD4-L82M afforded (S)-5 with TTNs comparable to iAMD4 (470 and 475, respectively) but with near-perfect enantioselectivity (>99\% ee).

Gratifyingly, these conditions could be applied readily to millimole-scale reactions. High isolated yields were obtained at 1$4 \mathrm{mmol}$ scales after a simple extraction of the reaction mixture with organic solvent followed by chromatographic purification (Figure 3B). Leftover starting material could be detected, whereas the nitrenoid precursor was fully consumed. We believe that complete substrate conversion is limited by catalyst deactivation during the course of the reaction.

For the iAMD8-catalyzed synthesis of $\mathbf{2 0}$, related nitrenoid precursors like 23 and 24 were also tolerated, with 2-4 fold reduced analytical yield but excellent product enantioselectivity (>99\% ee); $\mathbf{2 5}$ yielded only trace product (Figure $\mathbf{3 C}$ ).

The mechanistic landscape of enzymatic amidation was investigated using a combination of kinetic isotope effects (KIEs) and density functional theory (DFT) calculations. Non-competitive $k_{H} / k_{D}$ initial rate measurements for the amidation of ethylbenzene (3) and $d_{10}$-ethylbenzene (3-d $\left.\mathbf{d}_{10}\right)$ were carried out in side-by-side reactions using whole-cell transformations with the $i A M D 8$ variant A KIE of near unity $(1.06 \pm 0.02)$ is indicative that $\mathrm{C}-\mathrm{H}$ bond cleavage is not involved in the turnover-limiting step of the reaction (Figure 4). This value, and an observed first-order dependence of initial rates on the concentration of 4 , is consistent with nitrenoid formation as the turnover-limiting step in the enzymatic reaction. Nitrenoid formation being rate determining is a likely reason why the same enzyme variant displays divergent
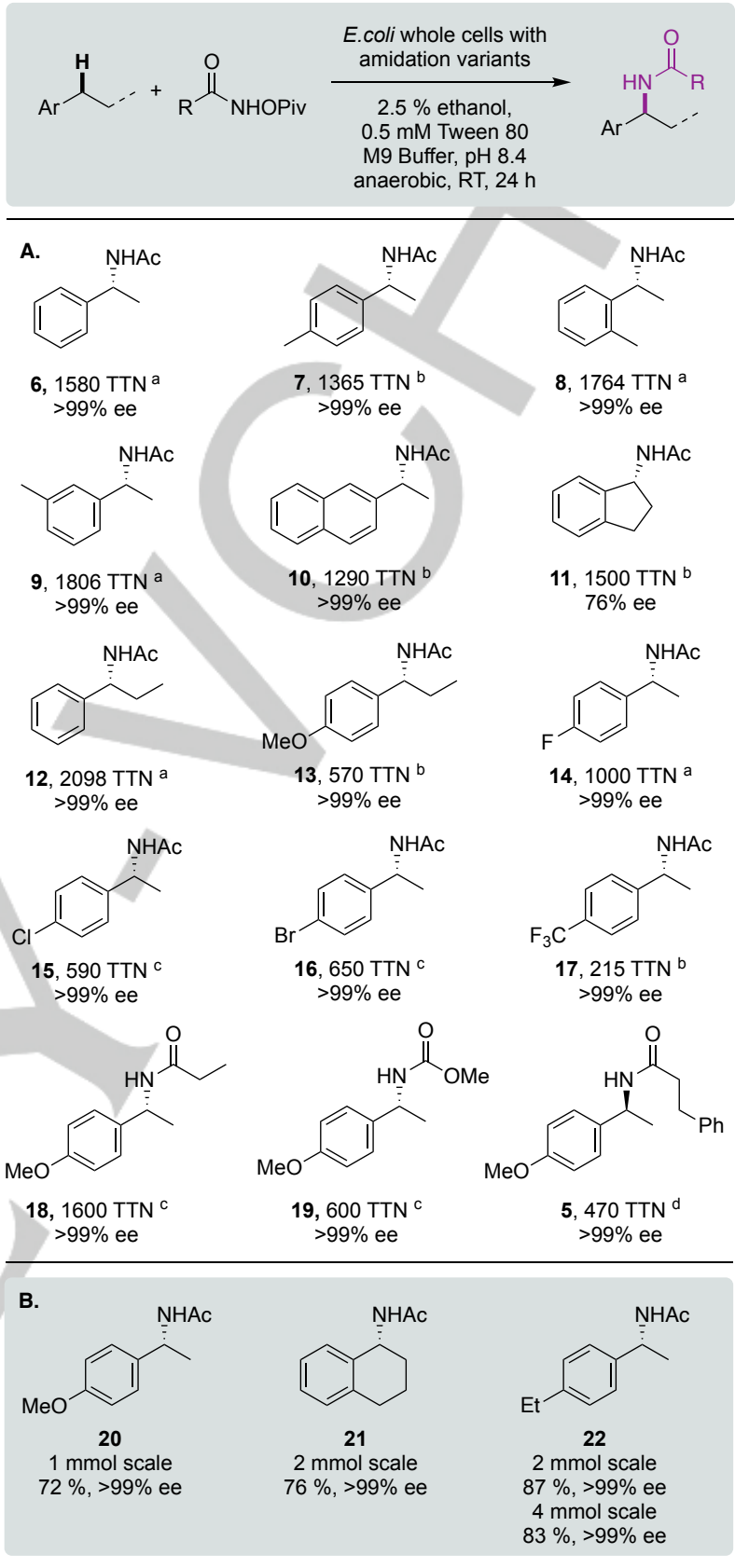

c.

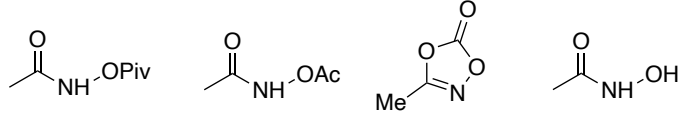

$$
\begin{aligned}
& \begin{array}{llll}
\mathbf{4}, 1.0 & \mathbf{2 3}, 0.4 & \mathbf{2 4}, 0.3 & \mathbf{2 5}, \text { trace }
\end{array}
\end{aligned}
$$

Figure 3: Substrate scope of benzylic amidation. (A) Analytical scale reactions performed with $5.0 \mathrm{mM}$ substrate and $10.0 \mathrm{mM}$ hydroxamate ester under wholecell conditions. "With iAMD9, "bith iAMD8, "with iAMD8b-A74T, d with iAMD4L82M. (B) Scaleup reactions performed with iAMD8-expressing E. coli cells. Yields refer to isolated product. (C) Relative analytical yield of 20 in iAMD8 catalyzed amidation with related nitrene precursors. Refer to Supplementary Information for experimental details.

reactivities with different nitrene precursors (see above). Competitive KIE experiments were utilized to investigate the $\mathrm{C}-\mathrm{H}$ functionalization step for amide formation with substrate $\mathbf{3}$, since selectivity and substrate scope depend on the nature of this 
step. ${ }^{[32,33]}$ A competitive KIE of $8.2 \pm 0.4$ indicates that this step involves $\mathrm{C}-\mathrm{H}$ bond cleavage. In previous heme-enzyme catalyzed nitrene $\mathrm{C}-\mathrm{H}$ insertion studies, large competitive KIE values have also been noted $\left(k_{H} / k_{D}=4.7,5.3\right)^{[34]}$, with the observation of a noncompetitive $\mathrm{KIE}$ greater than unity $\left(k_{H} / k_{\mathrm{D}}=2.7^{[34]}\right.$, $\left.1.6^{[35]}\right)$, indicating contribution of $\mathrm{C}-\mathrm{H}$ cleavage to the rate-determining step. These reports utilized azides as nitrenoid sources, which perhaps leads to facile nitrenoid formation.

For a quantitative interpretation of experimental KIEs, both concerted and stepwise pathways for $\mathrm{C}-\mathrm{H}$ bond functionalization were studied using DFT. Nitrenoid insertion was modeled with a truncated porphyrin system chelated to a methoxide group to simulate the serinate residue of iAMD8. Potential energy surface scans on all reactants were performed to map the conformational and configurational space for this reaction. A UB3LYP-D3BJ/6$31+G(d) / L A N L 2 D Z(F e)$ functional and basis set were utilized for geometry and transition-state optimizations. Single point energy corrections were calculated at the UB3LYP-D3BJ/6$311+G(d, p) / L A N L 2 T Z(f)$ level of theory and an implicit solvent model was applied (SMD-chlorobenzene) to simulate the dielectric of the enzyme pocket based on the benchmarks reported by Liu and Arnold. ${ }^{[35]}$ Attempts to locate transition states (TS) for concerted nitrenoid insertion on any of the spin-state surfaces were unsuccessful, suggesting that this is likely not a viable pathway for $\mathrm{C}-\mathrm{H}$ amidation. ${ }^{[35,36]}$ Stepwise nitrenoid insertion involving hydrogen atom transfer (HAT) followed by carbon-nitrogen bond formation via a radical rebound mechanism was then examined (Figure 5). An exploration of different spin states indicated that the lowest energy TS for HAT proceeds on the triplet surface (TS HAT-Triplet $_{\text {) }}$ while the radical rebound TS proceeds on the quintet surface (TS $\mathrm{CN}_{\mathrm{CN} \text {-Quintet). }}$ ).

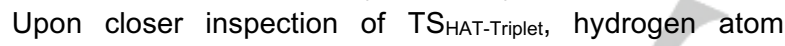
transfer is colinear and the hydrogen is equidistant from the nitrogen and benzylic carbon atoms. An iron-nitrogen distance of

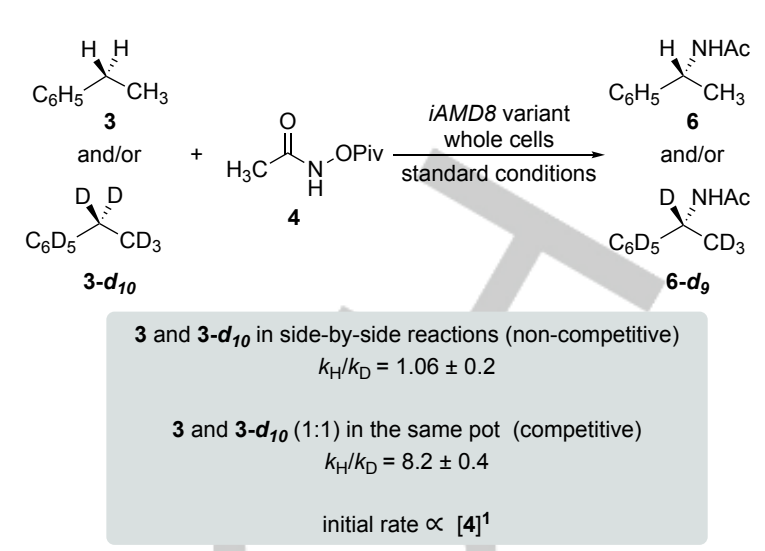

Figure 4: Kinetic studies of enzymatic amidation. Non-competitive KIE measurements performed in triplicate from independently determined rateconstants for $\mathbf{3}$ and $\mathbf{3}-\mathbf{d}_{10}$ carried out in side-by-side reactions. Competitive KIE measurements were performed from two batches of cells with 16 individual measurements of the ratio of products $\left(\mathbf{6}\right.$ and $\left.\mathbf{6}-\mathbf{d}_{9}\right)$ resulting from reaction of 3 and $\mathbf{3}-\mathbf{d}_{10}$ in the 'same pot'.

$1.94 \AA$ in combination with the electron density analysis of the nitrenoid connotes double bond-like character in TS HAT-Triplet. However, at the $\mathrm{C}-\mathrm{N}$ bond-forming step the iron-nitrogen bond elongates to $2.38 \AA$, indicating a switch to single-bond character

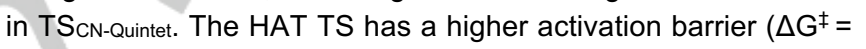
$22.0 \mathrm{kcal} / \mathrm{mol})$ than the radical rebound TS $\left(\Delta \mathrm{G}^{\ddagger}=17.6 \mathrm{kcal} / \mathrm{mol}\right)$, thereby making HAT the first irreversible step for product formation for ethyl benzene (Figure 5, see the supporting information for energetics of all electronic states). A theoretical KIE for HAT was calculated from TSHAT-triplet using the formalism of Bigeleisen and Mayer ${ }^{[37]}$ and including a Wigner tunneling correction; a theoretical $k_{H} / k_{D}$ value of 8.5 is in excellent agreement with the experimental value of $8.2 \pm 0.4$ (Figure 5).

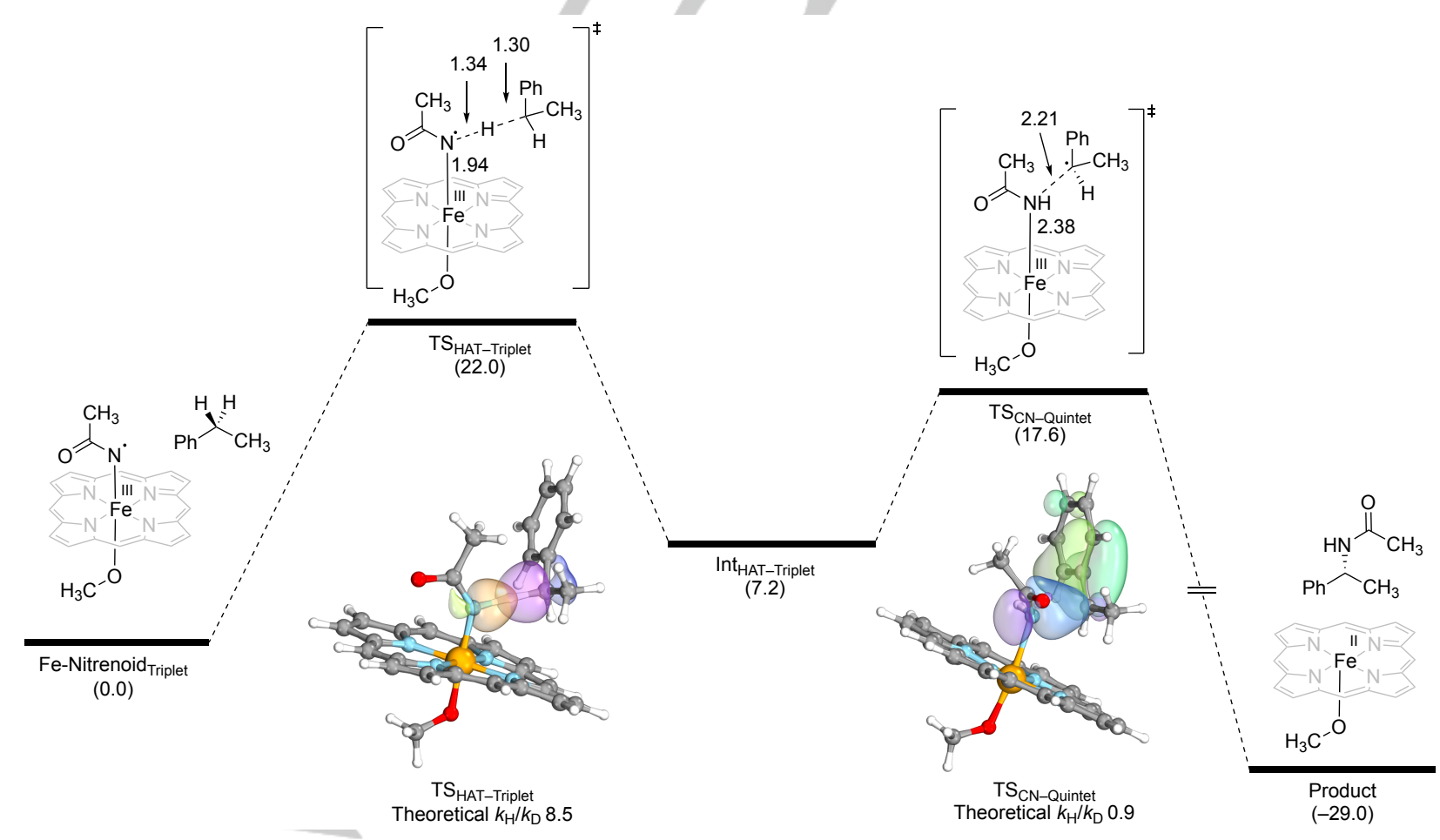

Figure 5: Potential energy surface of the porphyrin model system for stepwise HAT followed by radical rebound. An IBO analysis was used to depict

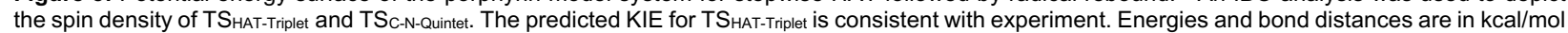
and angstroms respectively. 
Spin density computations along the reaction coordinate for HAT further point to the radical nature of the transferring hydrogen. In TS HAT-Triplet, the spin density on the nitrogen and benzylic carbon are each 0.5 , indicative of a concerted transfer of proton and electron as a hydrogen atom. Visualization of the spin density indicates this symmetric nature in the bonding orbitals; $\mathrm{C}-\mathrm{H}$ bond breaking is displayed as a purple orbital and $\mathrm{N}-\mathrm{H}$ bond formation is shown as an orange orbital (Figure 5, see the Supporting Information for the intrinsic bond orbital (IBO) analysis for the entire reaction coordinate). Following TS $\mathrm{S}_{\text {HAT-Triplet, the spin density }}$ on the benzylic carbon drops to 0.25 in IntHAT-Triplet indicating the delocalization of the radical $-a$ feature that is preserved in TS $\mathrm{C}_{\mathrm{N}-\mathrm{N}-}$ Quintet as evident from the IBOs shown as green orbitals. These results obtained from the porphyrin model system in conjunction with the deuterium KIEs provide insights into the chemical underpinnings of the enzymatic $\mathrm{C}-\mathrm{H}$ amidation reaction.

In conclusion, directed evolution of P411 enzymes has now enabled the intermolecular benzylic $\mathrm{C}-\mathrm{H}$ amidation of feedstock aromatic compounds, a transformation unreported with small molecule catalysts. Mechanistic studies reveal that nitrene formation from the hydroxamate precursors is the ratedetermining step in this enzymatic amidation process, with subsequent $\mathrm{C}-\mathrm{H}$ functionalization occuring via a stepwise HATradical rebound pathway. Diversifying the repertoire of biocatalytic chemistry requires accessing novel enzymes that can provide starting points for further evolution. This finding positions us to expand enzymatic amidation to more challenging substrates and to explore other reactions of acyl-nitrene species.

\section{Acknowledgements}

This research was supported in part by the NIH National Institute of General Medical Sciences (R01GM125887), the ACS GCI Pharmaceutical Roundtable Research Grant (F.H.A., S.V.A.), and Binghamton University startup funds (J.S.H.). J.S.H. and M.S.C. acknowledge support from the XSEDE Science Gateways Program (CHE180061 and CHE210031), which is supported by NSF grant number ACl-1548562. We thank S. Brinkmann-Chen for helpful discussion and comments. The content is solely the responsibility of the authors and does not necessarily represent the official views of the funding agencies.

Keywords: biocatalysis $\cdot$ nitrene transfer $•$ P411 enzymes • asymmetric $\mathrm{C}-\mathrm{H}$ functionalization

\section{References}

[1] H. Lundberg, F. Tinnis, N. Selander, H. Adolfsson, Chem. Soc. Rev. 2014, 43, 2714-2742.

[2] S. D. Roughley, A. M. Jordan, J. Med. Chem. 2011, 54, 3451-3479.

[3] M. T. Sabatini, Lee. T. Boulton, H. F. Sneddon, T. D. Sheppard, Nat Catal 2019, 2, 10-17.

[4] H. M. L. Davies, D. Morton, J. Org. Chem. 2016, 81, 343350.

[5] D. J. Abrams, P. A. Provencher, E. J. Sorensen, Chem. Soc. Rev. 2018, 47, 8925-8967.

[6] Y. Park, K. T. Park, J. G. Kim, S. Chang, J. Am. Chem. Soc. 2015, 137, 4534-4542.

[7] S. Y. Hong, Y. Park, Y. Hwang, Y. B. Kim, M.-H. Baik, S. Chang, Science 2018, 359, 1016-1021.

[8] Y. Park, Y. Kim, S. Chang, Chem. Rev. 2017, 117, 92479301.
[9] E. Tosi, R. M. de Figueiredo, J.-M. Campagne, Catalysts 2021, 11, 471.

[10] Y. Park, S. Chang, Nat. Catal. 2019, 2, 219-227.

[11] S. Y. Hong, D. Kim, S. Chang, Nat Catal 2021, 4, 79-88.

[12] E. Lee, Y. Hwang, Y. B. Kim, D. Kim, S. Chang, J. Am. Chem. Soc. 2021, jacs.1c02550.

[13] M. Ju, E. E. Zerull, J. M. Roberts, M. Huang, I. A. Guzei, J. M. Schomaker, J. Am. Chem. Soc. 2020, 142, 1293012936.

[14] H. Wang, Y. Park, Z. Bai, S. Chang, G. He, G. Chen, J. Am. Chem. Soc. 2019, 141, 7194-7201.

[15] Q. Xing, C.-M. Chan, Y.-W. Yeung, W.-Y. Yu, J. Am. Chem. Soc. 2019, 141, 3849-3853.

[16] Z. Zhou, S. Chen, Y. Hong, E. Winterling, Y. Tan, M. Hemming, K. Harms, K. N. Houk, E. Meggers, J. Am. Chem. Soc. 2019, 141, 19048-19057.

[17] L. Liu, H. Song, Y.-H. Liu, L.-S. Wu, B.-F. Shi, ACS Catal. 2020, 10, 7117-7122.

[18] W. Liu, W. Yang, J. Zhu, Y. Guo, N. Wang, J. Ke, P. Yu, C. $\mathrm{He}$, ACS Catal. 2020, 10, 7207-7215.

[19] S. Fukagawa, Y. Kato, R. Tanaka, M. Kojima, T. Yoshino, S. Matsunaga, Angew. Chem. Int. Ed. 2019, 58, 1153-1157.

[20] S. Fukagawa, M. Kojima, T. Yoshino, S. Matsunaga, Angew. Chem. Int. Ed. 2019, 58, 18154-18158.

[21] C. M. B. Farr, A. M. Kazerouni, B. Park, C. D. Poff, J. Won, K. R. Sharp, M.-H. Baik, S. B. Blakey, J. Am. Chem. Soc. 2020, 142, 13996-14004.

[22] O. F. Brandenberg, R. Fasan, F. H. Arnold, Curr. Opin. Biotechnol. 2017, 47, 102-111.

[23] Y. Yang, F. H. Arnold, Acc. Chem. Res. 2021, 54, 12091225.

[24] C. K. Prier, R. K. Zhang, A. R. Buller, S. Brinkmann-Chen, F. H. Arnold, Nat. Chem. 2017, 9, 629-634.

[25] Z.-J. Jia, S. Gao, F. H. Arnold, J. Am. Chem. Soc. 2020, 142, 10279-10283.

[26] I. Cho, C. K. Prier, Z. Jia, R. K. Zhang, T. Görbe, F. H. Arnold, Angew. Chem. Int. Ed. 2019, 58, 3138-3142.

[27] D. Li, T. Wu, K. Liang, C. Xia, Org. Lett. 2016, 18, 22282231.

[28] J. L. Jat, M. P. Paudyal, H. Gao, Q.-L. Xu, M. Yousufuddin, D. Devarajan, D. H. Ess, L. Kürti, J. R. Falck, Science 2014, 343, 61-65.

[29] Z. Ma, Z. Zhou, L. Kürti, Angew. Chem. Int. Ed. 2017, 56, 9886-9890.

[30] L. Legnani, G. Prina-Cerai, T. Delcaillau, S. Willems, B. Morandi, Science 2018, 362, 434-439.

[31] R. K. Zhang, K. Chen, X. Huang, L. Wohlschlager, H. Renata, F. H. Arnold, Nature 2019, 565, 67-72.

[32] E. M. Simmons, J. F. Hartwig, Angew. Chem. Int. Ed. 2012, 51, 3066-3072.

[33] J. Chan, A. Lewis, M. Gilbert, M.-F. Karwaski, A. J. Bennet, Nat. Chem. Biol. 2010, 6, 405-407.

[34] R. Singh, J. N. Kolev, P. A. Sutera, R. Fasan, ACS Catalysis 2015, 5, 1685-1691.

[35] Y. Yang, I. Cho, X. Qi, P. Liu, F. H. Arnold, Nat. Chem. 2019, 11, 987-993.

[36] J. Wang, H. Gao, L. Yang, Y. Q. Gao, ACS Catalysis 2020, $10,5318-5327$. Recent report by Gao and coworkers on intermolecular $\mathrm{C}-\mathrm{H}$ amination showing that the concerted nitrene insertion has a prohibitively high barrier on the singlet surface further supports our assertion that nitrene insertion is a stepwise pathway.

[37] J. Bigeleisen, M. G. Mayer, J. Chem. Phys. 1947, 15, 261267. 
WILEY-VCH

\section{Entry for the Table of Contents}

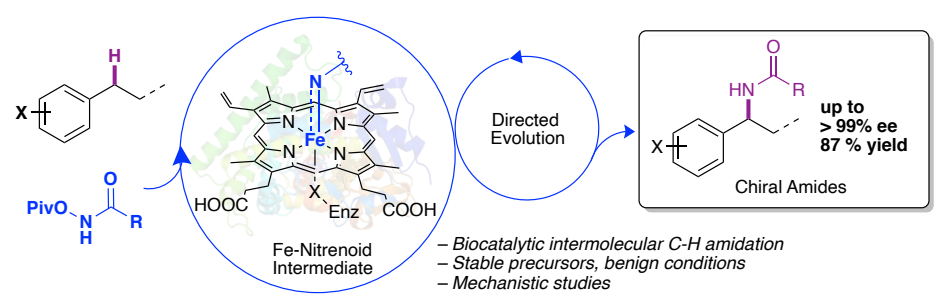

Directed evolution of P411 heme enzymes enables intermolecular $\mathrm{C}-\mathrm{H}$ amidation with high yields and exquisite enantioselectivity. The biocatalytic process utilizes stable hydroxamate esters as nitrenoid precursors and is amenable to scaleup. Mechanistic studies reveal rate-determining nitrenoid formation followed by a stepwise, hydrogen atom transfer-mediated $\mathrm{C}-\mathrm{H}$ functionalization. 